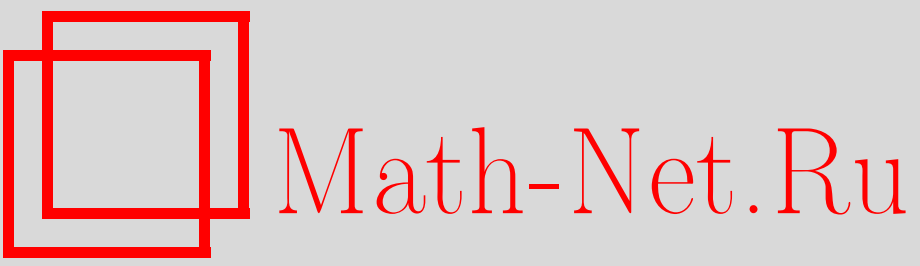

В. И. Кукулин, О. А. Рубцова, Формулировка квантовой теории рассеяния в терминах собственных дифференциалов (стационарных волновых пакетов), ТMФ, 2002, том 130, номер 1, 64-86

DOI: https://doi.org/10.4213/tmf291

Использование Общероссийского математического портала Math-Net.Ru подразумевает, что вы прочитали и согласны с пользовательским соглашением

http: //www. mathnet.ru/rus/agreement

Параметры загрузки:

IP : 54.80 .73 .141

26 апреля 2023 г., 14:20:57 
ТЕОРЕТИЧЕСКАЯ

И МАТЕМАТИЧЕСКАЯ

ФИЗИКА

Том 130, № 1

январь, 2002

(C) 2002 г.

В.И. Кукулин*, О.А. Рубцова*

\title{
ФОРМУЛИРОВКА КВАНТОВОЙ ТЕОРИИ РАССЕЯНИЯ В ТЕРМИНАХ СОБСТВЕННЫХ ДИФФЕРЕНЦИАЛОВ (СТАЦИОНАРНЫХ ВОЛНОВЫХ ПАКЕТОВ)
}

\begin{abstract}
Описывается построение основных операторов теории рассеяния на массовой поверхности и вне ее в терминах ограниченных в пространстве стационарных волновых пакетов или собственных дифференциалов. Использованный для этих целей метод опирается на определенную схему дискретизации континуума. Найденные впервые конечномерные аппроксимации для функции Грина и $T$-матрицы при любой энергии непосредственно строятся на основе простой однократной диагонализации матрицы гамильтониана на полном базисе $L_{2}$-типа. Показано, что развитый подход приводит к удобному конечномерному представлению операторов рассеяния в базисе волновых функций гармонического осциллятора. Метод допускает непосредственное обобщение на задачу рассеяния трех и большего числа тел.
\end{abstract}

\section{1. ВВЕДЕНИЕ}

Хорошо известно, что в квантовой теории изучение дискретного спектра (связанные состояния) и непрерывного спектра (состояния рассеяния) несопоставимы по сложности. Для исследования связанных состояний в квантовых системах, включающих десятки и сотни частиц, развиты весьма точные количественные методы (например, в квантовой химии молекул, а также многочисленные вариации метода Монте-Карло и других вариационных методов в ядерной физике и теории конденсированных сред). В задачах же многочастичного рассеяния аналогичные эффективные подходы отсутствуют, хотя обшая формулировка задачи рассеяния в системе $N$ частиц была дана много лет назад в работах Фаддеева и Якубовского (см. монографию [1], где приведены ссылки на оригинальные работы). Практическое решение задачи рассеяния уже для четырех частиц вызывает огромные сложности. Это резкое различие в сложности решения задач для дискретного и непрерывного спектров обусловлено принципиально разным поведением собственных волновых функций и разным типом граничных условий. В случае дискретного спектра все волновые функции экспоненциально спадают на больших расстояниях

${ }^{*}$ Научно-исследовательский институт ядерной физики, Московский государственный университет, Москва, Россия 
во всех направлениях в конфигурационном пространстве системы. Для непрерывного спектра точные волновые функции простираются до бесконечных расстояний и в многочастичных задачах отвечают нетривиальным граничным условиям [1]. Неудивительно поэтому, что наиболее распространенной схемой решения задач многочастичного рассеяния является так назьваемая кластерная редукция (метод связанных каналов [2]), при которой полная волновая функция многочастичного рассеяния аппроксимируется определенной суперпозицией конфигураций, отвечаюших связанным состояниям в подсистемах (кластерам) и относительному движению этих кластеров. При этом вместо очень сложного для изучения многочастичного континуума возникает более простая система связанных двухфрагментных каналов, которая уже допускает прямую численную реализацию в форме одномерных дифференциальных, интегральных или интегро-дифференциальных уравнений. Более внимательный анализ основных уравнений квантовой теории рассеяния, однако, показывает, что основные трудности в трактовке континуума можно обойти, если вместо точных волновых функций рассеяния использовать некоторые их средние (по энергии), так называемые собственные дифференциалы [3], которые локализованы в пространстве и отличаются (во внутренней области) от точных волновых функций рассеяния лишш конечной нормировкой. По своему физическому смыслу собственные дифференциалы аналогичны не зависящим от времени волновым пакетам [3]. Напомним, что в 30-е - 40-е годы зависящие от времени волновые пакеты были основным "инструментом" для изучения квантовой теории рассеяния.

Основное удобство при использовании языка собственных дифференциалов заключается, как показано в данной работе, в полной аналогии их свойств со свойствами векторов связанных состояний, что позволяет аппроксимировать их посредством конечной суммы квадратично-интегрируемых подходящих базисных функций, например, функций гармонического осциллятора. При этом для нахождения основных операторов теории рассеяния, таких как функция Грина и $T$-матрица, достаточно выполнить одну простую диагонализацию конечномерной матрицы гамильтониана на соответствующем $L_{2}$-базисе, т.е. фактически использовать тот же простой подход, что и для нахождения связанных состояний. Такой универсальный метод позволяет сушественно упростить решение задач теории рассеяния, особенно в случае комплексных и(или) нелокальных операторов взаимодействия.

Данная работа посвящена изложению обшего подхода и его применению к задачам двухчастичного рассеяния. В дальнейшем мы намерены обобшить данный подход на случай многочастичного рассеяния (некоторые характерные задачи в теории трехчастичного рассеяния, рассмотренные с помощью предлагаемого подхода, см. в работах $[4],[5])$.

\section{2. ПРЕДСТАВЛЕНИЕ СОСТОЯНИЙ РАССЕЯНИЯ ПОСРЕДСТВОМ СОБСТВЕННЫХ ДИФФЕРЕНЦИАЛОВ}

2.1. Собственные дифференциалы и их свойства. Рассмотрим некоторый гамильтониан $H$, описывающий движение частицы во внешнем центрально-симметричном поле (или взаимодействие двух частиц)

$$
H=H_{0}+V
$$

3 Теоретическая и математическая физика, т. 130, № 1, 2002 г. 
где $H_{0}$ - оператор кинетической энергии, $V$ - потенциал взаимодействия частицы с полем (или частиц между собой), который считается локальным или нелокальным.

Пусть $\{\Psi(E, l, m)\}$ обозначает полный набор состояний непрерывного спектра гамильтониана $H$, отвечаюший определенному значению орбитального момента $\hat{\mathbf{L}}^{2}$ и его проекции $L_{z}$ на ось $z$. Разобьем весь непрерывный спектр энергий $E \in(0,+\infty)$ на интервалы $E_{0}=0<E_{1}<\cdots<E_{n}<\cdots$ (в пределе $n \rightarrow \infty, E_{n} \rightarrow \infty$ ). Определим на каждом интервале собственньй дифференциал (С Д) радиальной части волновой функции $\Psi(E, l ; r)$ следуюшим образом:

$$
Y_{i}^{l}(r)=\frac{1}{\sqrt{\delta E_{i}}} \int_{E_{i-1}}^{E_{i}} \Psi(E, l ; r) d E,
$$

где $\delta E_{i}=E_{i}-E_{i-1}$. Легко показать, что СД $Y_{i}^{l}(r)$ обладают следующими свойствами:

$$
\begin{gathered}
\left\langle Y_{i}^{l}|H| Y_{j}^{l}\right\rangle=E_{i}^{*} \delta_{i, j}, \quad \text { где } E_{i}^{*}=\frac{1}{2}\left(E_{i}+E_{i-1}\right), \\
\left\langle Y_{i}^{l} \mid Y_{j}^{l}\right\rangle=\delta_{i, j} .
\end{gathered}
$$

Эти равенства являются прямым следствием определения (2), условия нормировки функций континуума $\Psi(E, l ; r)$ на $\delta$-функцию от энергии, а также того факта, что функции $\Psi(E, l ; r)$ являются собственными функциями гамильтониана $H$. Таким образом, функции $\left|Y_{i}^{l}\right\rangle$ представляют собой прямой аналог собственных функций гамильтониана $H$, отвечающих дискретному спектру.

Кроме того, можно доказать еше одно интересное свойство СД,

$$
\left\langle Y_{i}^{l}\left|H^{n}\right| Y_{j}^{l}\right\rangle=\left(E_{i}^{*}\right)^{n}\left[1+O\left(\left(\frac{\delta E_{i}}{E_{i}^{*}}\right)^{2}\right)\right] \delta_{i, j}, \quad n=2, \ldots,
$$

показывающее малость отличия $\left|Y_{i}^{l}\right\rangle$ от точных функций рассеяния.

Если в определении (2) разложить функцию $\Psi(E, l ; r)$ в ряд в окрестности точки $E=$ $E_{i}^{*}$, получается следующее соотношение:

$$
Y_{i}^{l}(r)=\sqrt{\delta E_{i}}\left(\Psi\left(E_{i}^{*}, l ; r\right)+O\left(\delta E_{i}^{2}\right)\right) .
$$

Очевидно, что последнее равенство будет вьполняться не при всех значениях $r$, а только в конечной области $r \leqslant R_{\max }$ ("на конечном носителе"). Для того чтобы яснее определить границу этой области, воспользуемся асимптотическим видом функции рассеяния $\Psi(E, l ; r)$ (при этом предполагается, что $R_{\max }$ лежит уже в асимптотической области),

$$
\Psi(E, l ; r) \Rightarrow \sqrt{\frac{2}{\pi k}} \frac{1}{2 i}\left(e^{-i\left(k r-\frac{l \pi}{2}\right)}-S_{l}(k) e^{i\left(k r-\frac{l \pi}{2}\right)}\right)
$$

Здесь $k=\sqrt{2 E}$ (в единицах $\hbar=m=1), S_{l}(k)$ - элементы $S$-матрицы.

Согласно определению (2) имеем

$$
Y_{i}^{l}(r)=\sqrt{\frac{2}{\pi}} \frac{1}{2 i} \frac{1}{\sqrt{\delta E_{i}}} \int_{E_{i-1}}^{E_{i}} d k \sqrt{k}\left(e^{-i\left(k r-\frac{l \pi}{2}\right)}-S_{l}(k) e^{i\left(k r-\frac{l \pi}{2}\right)}\right) .
$$


Будем считать, что $S_{l}(k)$ меняется на отрезке $\left(E_{i-1}, E_{i}\right)$ гораздо медленнее, чем $e^{i k r}$ (равно как и функция $\sqrt{k}$ ), что всегда справедливо при больших $r$. Тогда, интегрируя и упрощая $(6 \mathrm{a})$, получим

$$
Y_{i}^{l}(r) \approx \Psi\left(E_{i}^{*}, l ; r\right) \sqrt{\delta E_{i}} \frac{\sin \left(\frac{\delta k_{i}}{2} r\right)}{\frac{\delta k_{i}}{2} r}, \quad \delta k_{i}=\frac{\delta E_{i}}{\sqrt{2 E_{i}^{*}}} .
$$

Поскольку множитель $\sin \left(\delta k_{i} r / 2\right) /\left(\delta k_{i} r / 2\right) \approx 1$ на расстояниях $r$, удовлетворяющих условию $\delta k_{i} r / 2 \ll 1$, можно заключить, что "на конечном носителе" СД отличаются от точных функций рассеяния только нормировочным множителем, т.е.

$$
Y_{i}^{l}(r)=\sqrt{\delta E_{i}} \Psi\left(E_{i}^{*}, l ; r\right) \quad \text { при } r \ll R_{\max }^{i} .
$$

Граница “конечного носителя" $R_{\max }^{i}$ определяется естественным соотношением

$$
R_{\max }^{i}=\frac{\sqrt{8 E_{i}^{*}}}{\delta E_{i}}
$$

За этой границей функция $Y_{i}^{l}(r)$ быстро спадает, что и обеспечивает ее конечную нормировку.

Случай резонанса следует оговорить особо. Если резонанс достаточно узкий, $S$-матрица меняется резко на интервале $\delta E_{i}$, однако радиальная волновая функция в точке резонанса быстро уменшшается вне области взаимодействия и начинает медленно возрастать только на очень больших расстояниях. При этом соотношение (8) сохраняется (может поменяться лишь нормировочный множитель), поскольку СД будет совпадать с радиальной волновой функцией при $r \ll R_{\max }$, где $R_{\max } \approx 1 / \sqrt{\Gamma}$ (Г- ширина резонанса). Если же резонанс широкий, то $S$-матрица меняется весьма плавно на достаточно узком интервале $\delta E_{i}$ и наши рассуждения остаются в силе.

Сам процесс замены точных собственных функций непрерывного спектра $H$ на их дискретные аналоги является по существу дискретизацией континуума. Важно подчеркнуть, что обычное математически корректное построение квантовой механики основано на векторах гильбертова пространства (см., например, [3]). Даже определение эрмитовости операторов дается обычно в терминах векторов гильбертова пространства. Очевидно, что векторы $\left|Y_{i}^{l}\right\rangle$ являются квадратично-интегрируемыми даже в предельном случае $\delta E_{i} \rightarrow 0$. Основная цель настоящего исследования состоит в адекватной замене функции Грина и $T$-матрицы, вычисленных по точным функциям континуума, на их дискретные аналоги.

2.2. Разложение единичного оператора. Система точных функций рассеяния $\{\Psi(E, l ; r)\}$ обладает полнотой в некотором функциональном пространстве $\mathcal{H}$, что можно символически записать в виде

$$
\mathbf{1}_{\mathcal{H}}^{l}=\int_{0}^{\infty} d E|\Psi(E, l)\rangle\langle\Psi(E, l)| .
$$

Ядро единичного оператора $\mathbf{1}_{\mathcal{H}}^{l}$ можно представить как сумму

$$
I^{l}\left(r, r^{\prime}\right)=\int_{0}^{\infty} d E \Psi(E, l ; r) \Psi^{*}\left(E, l ; r^{\prime}\right) \equiv \sum_{i=1}^{\infty} I_{i}^{l}\left(r, r^{\prime}\right),
$$


где

$$
I_{i}^{l}\left(r, r^{\prime}\right)=\int_{E_{i-1}}^{E_{i}} d E \Psi(E, l ; r) \Psi^{*}\left(E, l ; r^{\prime}\right)
$$

Действуя аналогично в дискретизованном континууме, из системы СД $\left\{\left|Y_{i}^{l}\right\rangle\right\}$ можно составить спектральную сумму

$$
\widehat{P}=\sum_{i=1}^{\infty}\left|Y_{i}^{l}\right\rangle\left\langle Y_{i}^{l}\right|
$$

причем ядро последнего оператора естественным образом может быть записано в виде

$$
P_{l}\left(r, r^{\prime}\right) \equiv \sum_{i=1}^{\infty} \hat{I}_{i}^{l}\left(r, r^{\prime}\right)
$$

где

$$
\hat{I}_{i}^{l}\left(r, r^{\prime}\right)=\frac{1}{\delta E_{i}} \int_{E_{i-1}}^{E_{i}} d E \Psi(E, l ; r) \int_{E_{i-1}}^{E} d E \Psi^{*}\left(E, l ; r^{\prime}\right)
$$

(чтобы различать операторы в дискретизованном представлении, будем снабжать их сверху “шляпкой"). Если разложить, как и в п. 2.1, подынтегральные выражения (12) и (13) в ряд в окрестности точки $E=E_{i}^{*}$, получим приближенное равенство

$$
I_{i}^{l}\left(r, r^{\prime}\right) \approx \hat{I}_{i}^{l}\left(r, r^{\prime}\right) \approx \Psi\left(E_{i}^{*}, l ; r\right) \Psi^{*}\left(E_{i}^{*}, l ; r^{\prime}\right) \delta E_{i},
$$

которое опять будет справедливо "на конечном носителе". Легко показать, используя асимптотический вид функций рассеяния, что равенство (14) верно при условиях

$$
r \ll R_{\max }^{i}, \quad r^{\prime} \ll R_{\max }^{i},
$$

где $R_{\max }^{i}$ определяется соотношением (9). Таким образом, можно заключить, что

$$
\begin{gathered}
I^{l}\left(r, r^{\prime}\right) \approx P^{l}\left(r, r^{\prime}\right) \quad \text { при } r, r^{\prime} \ll R_{\max } \\
R_{\max }=\min _{i} R_{\max }^{i} .
\end{gathered}
$$

Если теперь взять произвольную квадратично-интегрируемую функцию $\left|\Phi_{0}\right\rangle$ из пространства $\mathcal{H}$, характерный радиус которой меньше $R_{\max }$, то будет выполняться равенстBO

$$
\left\langle\Phi_{0} \mid \Phi_{0}\right\rangle=\left\langle\Phi_{0}\left|\mathbf{1}_{\mathcal{H}}^{l}\right| \Phi_{0}\right\rangle=\left\langle\Phi_{0}|\widehat{P}| \Phi_{0}\right\rangle
$$

Поскольку при $\delta E_{i} \rightarrow 0 R_{\max } \rightarrow \infty$, последнее равенство будет справедливо в этом пределе для широкого класса $L_{2}$-функций $\left|\Phi_{0}\right\rangle$.

Отсюда можно сделать вывод, что дискретный набор состояний $\left\{\left|Y_{i}^{l}\right\rangle\right\}_{i=1}^{\infty}$ будет обладать квазиполнотой в подпространстве квадратично-интегрируемых функций пространства $\mathcal{H}$ при достаточно малых ширинах $\delta E_{i}$. 
2.3. Спектральное разложение оператора Грина в представлении СД. Пусть теперь $\{\Psi(E, l m)\}$ - набор состояний непрерывного спектра, а $\left\{\Psi_{n, l m}\right\}$ - набор состояний дискретного спектра гамильтониана (1). Причем вместе эти функции образуют полную систему, т.е.

$$
1=\sum_{l m}\left\{\sum_{n}\left|\Psi_{n, l m}\right\rangle\left\langle\Psi_{n, l m}\left|+\int d E\right| \Psi(E, l m)\right\rangle\langle\Psi(E, l m)|\right\} .
$$

Тогда для точного оператора Грина гамильтониана $H$

$$
G^{(+)}(E)=[H-(E+i 0)]^{-1}
$$

можно воспользоваться хорошо известным спектральным разложением

$$
G^{(+)}(E)=\sum_{l m}\left\{\sum_{n} \frac{\left|\Psi_{n, l m}\right\rangle\left\langle\Psi_{n, l m}\right|}{E_{n, l}-E}+\int d E^{\prime} \frac{\left|\Psi\left(E^{\prime}, l m\right)\right\rangle\left\langle\Psi\left(E^{\prime}, l m\right)\right|}{E^{\prime}-(E+i 0)}\right\} .
$$

Выделим теперь парциальные функции Грина и запишем их в координатном представлении,

$$
G_{l}^{(+)}\left(E ; r, r^{\prime}\right)=\sum_{n} \frac{\Psi_{n, l}(r) \Psi_{n, l}\left(r^{\prime}\right)}{E_{n, l}-E}+\int d E^{\prime} \frac{\Psi\left(E^{\prime}, l ; r\right) \Psi\left(E^{\prime}, l ; r^{\prime}\right)}{E^{\prime}-(E+i 0)} .
$$

В последнее разложение входят радиальные части волновых функций, отвечающих орбитальному моменту $l$. Выделим в соотношении (20) действительную и мнимую части,

$$
\begin{gathered}
\operatorname{Re} G_{l}^{(+)}\left(E ; r, r^{\prime}\right)=\sum_{n} \frac{\Psi_{n, l}(r) \Psi_{n, l}\left(r^{\prime}\right)}{E_{n, l}-E}+\mathcal{P} \int d E^{\prime} \frac{\Psi\left(E^{\prime}, l ; r\right) \Psi\left(E^{\prime}, l ; r^{\prime}\right)}{E^{\prime}-E}, \\
\operatorname{Im} G_{l}^{(+)}\left(E ; r, r^{\prime}\right)=i \pi \Psi(E, l ; r) \Psi\left(E, l ; r^{\prime}\right) .
\end{gathered}
$$

Будем считать,что весь непрерывный спектр энергий разбит на полосы как в п. 2.1. Предполагается,что в дальнейшем нам потребуются матричные элементы оператора Грина $G_{l}^{(+)}$между некоторыми квадратично-интегрируемыми функциями. Целью этого пункта является представление функции $G_{l}^{(+)}$не через точные собственные функции непрерывного спектра $H$, как в равенствах (21) и (22), а через их собственные дифференциалы (поэтому достаточно аппроксимировать ядро оператора Грина в конечной области, определяемой (17)).

Рассмотрим сначала вклад состояний непрерьвного спектра в действительную часть функции Грина (21). Введем следующие обозначения:

$$
\mathcal{P} \int d E^{\prime} \frac{\Psi\left(E^{\prime}, l ; r\right) \Psi\left(E^{\prime}, l ; r^{\prime}\right)}{E^{\prime}-E}=\sum_{i=1}^{\infty} F_{i}(E)
$$

где

$$
F_{i}(E)=\mathcal{P} \int_{E_{i-1}}^{E_{i}} d E^{\prime} \frac{\Psi\left(E^{\prime}, l ; r\right) \Psi\left(E^{\prime}, l ; r^{\prime}\right)}{E^{\prime}-E}
$$


т.е. величина $F_{i}(E)$ определяет точный вклад в спектральный интеграл от энергетической полосы $\left[E_{i-1}, E_{i}\right]$ в непрерывном спектре. Здесь разбиение спектра такое же, как и в п. 2.1. Разложим билинейную форму, составляющую числитель подынтегрального выражения в полосе $\delta E_{i}$, в ряд по степеням $\left(E^{\prime}-E_{i}^{*}\right)$,

$$
\begin{aligned}
\Psi\left(E^{\prime}, l ; r\right) \Psi\left(E^{\prime}, l ; r^{\prime}\right)= & \Psi\left(E_{i}^{*}, l ; r\right) \Psi\left(E_{i}^{*}, l ; r^{\prime}\right)+ \\
& +\left[\frac{\partial\left[\Psi(E, l ; r) \Psi\left(E, l ; r^{\prime}\right)\right]}{\partial E}\right]_{E=E_{i}^{*}}\left(E^{\prime}-E_{i}^{*}\right)+\cdots
\end{aligned}
$$

Тогда

$$
F_{i}(E)=\sum_{k=0}^{\infty} \frac{1}{k !}\left[\frac{\partial^{k}\left[\Psi(E, l ; r) \Psi\left(E, l ; r^{\prime}\right)\right]}{\partial E^{k}}\right]_{E=E_{i}^{*}} J_{i}^{k}(E),
$$

где

$$
J_{i}^{k}=\int_{E_{i-1}}^{E_{i}} d E^{\prime} \frac{\left(E^{\prime}-E_{i}^{*}\right)^{k}}{E^{\prime}-E}, \quad k=0, \ldots
$$

Все интегралы (27) регулярны, если только значение энергии $E$ не совпадает с узлами разбиения $E_{i}$.

В дальнейшем нам понадобятся лишь первые два слагаемых ряда (26), для которых

$$
J_{i}^{0}=\ln \left|\frac{E_{i}-E}{E_{i-1}-E}\right|, \quad J_{i}^{1}=\delta E_{i}+\left(E-E_{i}^{*}\right) \ln \left|\frac{E_{i}-E}{E_{i-1}-E}\right| .
$$

Вообще же можно показать, что

$$
J_{i}^{k}=\left(\delta E_{i}\right)^{k} \tilde{J}_{i}^{k}\left(\frac{E-E_{i}^{*}}{\delta E_{i}}\right)
$$

где $\tilde{J}_{i}^{k}$ - ряд, включающий либо положительные степени аргумента (при $E \in$ $\left.\left(E_{i-1}, E_{i}\right)\right)$, либо отрицательные степени аргумента (при $\left.E \notin\left(E_{i-1}, E_{i}\right)\right)$.

Итак, имеем

$$
F_{i}(E)=\sum_{k=0}^{\infty} \frac{1}{k !}\left[\frac{\partial^{k}\left[\Psi(E, l ; r) \Psi\left(E, l ; r^{\prime}\right)\right]}{\partial E^{k}}\right]_{E=E_{i}^{*}}\left(\delta E_{i}\right)^{k} \tilde{J}_{i}^{k}\left(\frac{E-E_{i}^{*}}{\delta E_{i}}\right)
$$

Можно записать аналогичное (25) разложение для нахождения мнимой части функции Грина,

$$
\begin{aligned}
& \operatorname{Im} G_{l}^{(+)}\left(E ; r, r^{\prime}\right)=i \pi\left\{\Psi\left(E_{j}^{*}, l ; r\right) \Psi\left(E_{j}^{*}, l ; r^{\prime}\right)+\right. \\
& \left.\quad+\left[\frac{\partial\left[\Psi(E, l ; r) \Psi\left(E, l ; r^{\prime}\right)\right]}{\partial E}\right]_{E=E_{j}^{*}} \delta E_{j} \frac{\left(E^{\prime}-E_{i}^{*}\right)}{\delta E_{j}}+\cdots\right\}, \quad E \in\left(E_{j-1}, E_{j}\right) .
\end{aligned}
$$


Рассмотрим произведение $Y_{i}^{l}(r) Y_{i}^{l}\left(r^{\prime}\right)=\hat{I}_{i}^{l}\left(r, r^{\prime}\right)$ на интервале $\left(E_{i-1}, E_{i}\right)($ см. $(13))$. Разложив в ряд каждое подынтегральное выражение и проинтегрировав, запишем

$$
\begin{aligned}
Y_{i}^{l}(r) Y_{i}^{l}\left(r^{\prime}\right)= & \Psi\left(E_{i}^{*}, l ; r\right) \Psi\left(E_{i}^{*}, l ; r^{\prime}\right) \delta E_{i}+\frac{1}{2}\left(\Psi\left(E_{i}^{*}, l ; r\right)\left[\frac{\partial^{2} \Psi\left(E, l ; r^{\prime}\right)}{\partial E^{2}}\right]_{E=E_{i}^{*}}+\right. \\
& \left.+\Psi\left(E_{i}^{*}, l ; r^{\prime}\right)\left[\frac{\partial^{2} \Psi(E, l ; r)}{\partial E^{2}}\right]_{E=E_{i}^{*}}\right)\left(\delta E_{i}\right)^{3} \frac{1}{12}+O\left(\left(\delta E_{i}\right)^{5}\right) .
\end{aligned}
$$

Можем условно считать, что в конечной области $r<R_{\max }$ (см. (17))

$$
\Psi\left(E_{i}^{*}, l ; r\right) \Psi\left(E_{i}^{*}, l ; r^{\prime}\right)=\frac{Y_{i}^{l}(r) Y_{i}^{l}\left(r^{\prime}\right)}{\delta E_{i}}+O\left(\left(\delta E_{i}\right)^{2}\right) .
$$

Отсюда можно видеть, что при замене в разложениях (29) и (30) точных функций рассеяния их дискретными аналогами согласно равенству (32) не имеет смысла учитывать более двух первых слагаемых, так как третьи слагаемые в каждом из рядов (29) и (30) имеют тот же порядок малости $O\left(\left(\delta E_{i}\right)^{2}\right)$, что и погрешности двух первых слагаемых.

Для дискретной аппроксимации нам понадобится еще аналог билинейной формы

$$
\left[\frac{\partial \Psi(E, l ; r) \Psi\left(E, l, r^{\prime}\right)}{\partial E}\right]_{E=E_{i}^{*}} .
$$

Запишем аналогичное (31) разложение для $Y_{i+1}^{l}$,

$$
\begin{aligned}
Y_{i+1}^{l}(r) Y_{i+1}^{l}\left(r^{\prime}\right)=\delta & E_{i+1}\left\{\Psi\left(E_{i}^{*}, l ; r\right) \Psi\left(E_{i}^{*}, l ; r^{\prime}\right)+\right. \\
& \left.+\left[\frac{\partial \Psi(E, l ; r) \Psi\left(E, l, r^{\prime}\right)}{\partial E}\right]_{E=E_{i}^{*}}\left(E_{i+1}^{*}-E_{i}^{*}\right)+O\left(\left(\delta E_{i}\right)^{2}\right)\right\} .
\end{aligned}
$$

Рассмотрим далее разность

$$
\begin{gathered}
\frac{Y_{i+1}^{l}(r) Y_{i+1}^{l}\left(r^{\prime}\right)}{\delta E_{i+1}}-\frac{Y_{i}^{l}(r) Y_{i}^{l}\left(r^{\prime}\right)}{\delta E_{i}}=\left[\frac{\partial \Psi(E, l ; r) \Psi\left(E, l, r^{\prime}\right)}{\partial E}\right]_{E=E_{i}^{*}} \delta E_{i}+ \\
+\left[\frac{\partial \Psi(E, l ; r) \Psi\left(E, l, r^{\prime}\right)}{\partial E}\right]_{E=E_{i}^{*}} \frac{\delta E_{i+1}-\delta E_{i}}{2}+O\left(\left(\delta E_{i}\right)^{2}\right) .
\end{gathered}
$$

Второе слагаемое в правой части последнего выражения пропорционально $\delta E_{i+1}-\delta E_{i}$. Эта поправка связана с отличием взятого нами разбиения от эквидистантного. Очевидно, что в локальном смысле эти отличия невелики и, следовательно, можно считать, что второе слагаемое также имеет порядок малости $O\left(\left(\delta E_{i}\right)^{2}\right)$ (здесь имеется в виду не произвольное разбиение всего непрерьвного спектра на конечные полосы, а квазидискретный спектр, получаемый диагонализацией матрицы гамильтониана на конечном $L_{2}$-базисе (см. раздел 3$)$ ).

Итак, запишем, наряду с (32), конечномерную аппроксимацию и для производных,

$$
\left[\frac{\partial \Psi(E, l ; r) \Psi\left(E, l, r^{\prime}\right)}{\partial E}\right]_{E=E_{i}^{*}} \delta E_{i}=\frac{Y_{i+1}^{l}(r) Y_{i+1}^{l}\left(r^{\prime}\right)}{\delta E_{i+1}}-\frac{Y_{i}^{l}(r) Y_{i}^{l}\left(r^{\prime}\right)}{\delta E_{i}}+O\left(\left(\delta E_{i}\right)^{2}\right) .
$$


Заметим также, что в случае эквидистантного разбиения будет иметь место соотношение

$$
\begin{aligned}
& {\left[\frac{\partial \Psi(E, l ; r) \Psi\left(E, l, r^{\prime}\right)}{\partial E}\right]_{E=E_{i}^{*}} \delta E_{i}=} \\
& \quad=\frac{1}{2}\left\{\frac{Y_{i+1}^{l}(r) Y_{i+1}^{l}\left(r^{\prime}\right)}{\delta E_{i+1}}-\frac{Y_{i-1}^{l}(r) Y_{i-1}^{l}\left(r^{\prime}\right)}{\delta E_{i-1}}\right\}+O\left(\left(\delta E_{i}\right)^{3}\right) .
\end{aligned}
$$

Теперь можно привести окончательную формулу для конечномерной аппроксимации функции Грина в виде разложения по С Д точных функций рассеяния (или, выражаясь кратко, в представлении С Д (ПСД)),

$$
\begin{aligned}
G_{l}^{(+)}(E) \approx & \sum_{n} \frac{\left|\Psi_{n, l}\right\rangle\left\langle\Psi_{n, l}\right|}{E_{n, l}-E}+\sum_{i=1}\left\{\frac{\left|Y_{i}^{l}\right\rangle\left\langle Y_{i}^{l}\right|}{\delta E_{i}} \ln \left|\frac{E_{i}-E}{E_{i-1}-E}\right|+\right. \\
& \left.+\left(\frac{\left|Y_{i+1}^{l}\right\rangle\left\langle Y_{i+1}^{l}\right|}{\delta E_{i+1}}-\frac{\left|Y_{i}^{l}\right\rangle\left\langle Y_{i}^{l}\right|}{\delta E_{i}}\right)\left[1+\frac{\left(E-E_{i}^{*}\right)}{\delta E_{i}} \ln \left|\frac{E_{i}-E}{E_{i-1}-E}\right|\right]\right\}+ \\
& +i \pi\left\{\frac{\left|Y_{j}^{l}\right\rangle\left\langle Y_{j}^{l}\right|}{\delta E_{j}}+\left(\frac{\left|Y_{j+1}^{l}\right\rangle\left\langle Y_{j+1}^{l}\right|}{\delta E_{j+1}}-\frac{\left|Y_{j}^{l}\right\rangle\left\langle Y_{j}^{l}\right|}{\delta E_{j}}\right) \frac{\left(E-E_{j}^{*}\right)}{\delta E_{j}}\right\}
\end{aligned}
$$

при $E \in\left(E_{j-1}, E_{j}\right)$. Как следует из предыдушего вывода, ядро этого оператора будет совпадать с ядром точного оператора Грина в конечной области (17).

Следует отметить, что третье слагаемое в действительной части (37) является поправкой ко второму вне сингулярного интервала и играет, как можно явно показать, сушественную роль на этом интервале.

Итак, найденное здесь конечномерное представление для функции Грина (37) вместо неограниченных в пространстве точных функций рассеяния включает локализованные С Д, которые легко находятся путем разложения по любому полному $L_{2}$-базису (см. ниже раздел 3$)$.

2.4. Матрица рассеяния в базисе СД функций свободного движения. Определим согласно [6] оператор, отвечаюший рассеянию частицы на потенциале $V$,

$$
T(E)=V-V G^{(+)}(E) V,
$$

где $G^{(+)}$- оператор Грина гамильтониана (1). Элемент обобщенной $T$-матришы вне энергетической поверхности имеет вид

$$
T_{l l^{\prime}}\left(E_{1}, E_{2} ; E\right)=\left\langle\Psi_{0}\left(E_{1}, l\right)|T(E)| \Psi_{0}\left(E_{2}, l^{\prime}\right)\right\rangle .
$$

В последнем равенстве $\left|\Psi_{0}(E, l)\right\rangle$ - парциальная волновая функция свободного движения, нормированная на $\delta$-функцию по энергии. Если потенциал не содержит тензорной компоненты, $T$-матрица является диагональным по $l$ оператором,

$$
T_{l l^{\prime}}\left(E_{1}, E_{2} ; E\right)=T_{l}\left(E_{1}, E_{2} ; E\right) \delta_{l, l^{\prime}} .
$$


Найдем теперь выражение для $T_{l}\left(E_{1}, E_{2} ; E\right)$, используя вместо точных функций $\left|\Psi_{0}(E, l)\right\rangle$ их С. Р. Рассмотрим некоторое разбиение на интервалы непрерывного спектра гамильтониана свободного движения $H_{0}$,

$$
0=\mathcal{E}_{0}<\mathcal{E}_{1}<\cdots<\mathcal{E}_{i}<\cdots, \quad \Delta_{i}=\mathcal{E}_{i}-\mathcal{E}_{i-1}, \quad \mathcal{E}_{i}^{*}=\frac{1}{2}\left(\mathcal{E}_{i}+\mathcal{E}_{i-1}\right) .
$$

Построим на каждом интервале СД от функции свободного движения

$$
X_{i}^{l}(r)=\frac{1}{\sqrt{\Delta_{i}}} \int_{\mathcal{E}_{i-1}}^{\mathcal{E}_{i}} \Psi_{0}(E, l ; r) d E .
$$

Разлагая подынтегральное выражение в $(42)$ в ряд по степеням $\left(E-E_{i}^{*}\right)$ и упрошая, получим соотношение, аналогичное (5),

$$
\Psi_{0}\left(\mathcal{E}_{i}^{*}, l ; r\right)=\frac{X_{i}^{l}(r)}{\sqrt{\Delta_{i}}}+O\left(\Delta_{i}^{2}\right) .
$$

Еще раз подчеркнем, что подобное равенство имеет смысл внутри конечной гиперсферы $\left(r<R_{\max }^{0}, R_{\max }^{0}=\min \left(\sqrt{8 \mathcal{E}_{i}} / \Delta_{i}\right)\right)$ в конфигурационном пространстве.

Таким образом, для элемента внемассовой матрицы рассеяния получаем соотношение

$$
T_{l}\left(\mathcal{E}_{i}^{*}, \mathcal{E}_{j}^{*} ; E\right) \equiv T_{i, j}^{l}(E) \approx \frac{1}{\sqrt{\Delta_{i} \Delta_{j}}}\left\{\left\langle X_{i}^{l}|V| X_{j}^{l}\right\rangle-\left\langle X_{i}^{l}\left|V G_{l}^{(+)}(E) V\right| X_{j}^{l}\right\rangle\right\} .
$$

Если потенциал взаимодействия $V$ имеет конечный радиус действия $R$, то при достаточно малых ширинах энергетических полос $\Delta_{i}$, таких, что соответствуюший размер волновых пакетов $X_{i}^{l}(r) R_{\max }^{0}>R$, аппроксимация (44) должна давать практически точный результат. Выражение (44) для $T_{l}$ мы будем называть матрицей рассеяния в ПСД.

Выведем еше одно важное соотношение. По аналогии с (30) можно записать интерполяционную формулу для волновой функции свободного движения в виде

$$
\Psi_{0}(E, l ; r) \approx \frac{X_{j}^{l}(r)}{\sqrt{\Delta_{j}}}+\left(\frac{X_{j+1}^{l}(r)}{\sqrt{\Delta_{j+1}}}-\frac{X_{j}^{l}(r)}{\sqrt{\Delta_{j}}}\right) \frac{\left(E-\mathcal{E}_{j}^{*}\right)}{\Delta_{j}} \quad \text { при } \quad E \in\left(\mathcal{E}_{j-1}, \mathcal{E}_{j}\right)
$$

Поэтому для нахождения "конечномерной” функции $T_{l}$ вне массовой поверхности в ПСД можно использовать более обшую формулу, в которой оставлены только линейные по $\left(E-\mathcal{E}^{*}\right) / \Delta$ члены,

$$
\begin{aligned}
T_{l}\left(E_{1}, E_{2} ; E\right) \approx & T_{i, j}^{l}(E)+\left(T_{i+1, j}^{l}(E)-T_{i, j}^{l}(E)\right) \frac{\left(E_{1}-\mathcal{E}_{i}^{*}\right)}{\Delta_{i}}+ \\
& +\left(T_{i, j+1}^{l}(E)-T_{i, j}^{l}(E)\right) \frac{\left(E_{2}-\mathcal{E}_{j}^{*}\right)}{\Delta_{j}}
\end{aligned}
$$

при $E_{1} \in\left(\mathcal{E}_{i-1}, \mathcal{E}_{i}\right), E_{2} \in\left(\mathcal{E}_{j-1}, \mathcal{E}_{j}\right)$. Эта интерполящионная формула для внемассовой $T$-матрицы должна быть весьма точной при достаточно малых ширинах энергетических полос $\Delta_{i}(i=1, \ldots)$. 
Далее, найдем матричные элементы $T_{i, j}^{l}(E)$, подставляя в $(44)$ выражение для $G_{l}^{(+)}(E)$. Обозначим

$$
V_{i j} \equiv \frac{\left\langle X_{i}^{l}|V| X_{j}^{l}\right\rangle}{\sqrt{\Delta_{i} \Delta_{j}}}, \quad \tilde{V}_{i k} \equiv \frac{\left\langle X_{i}^{l}|V| Y_{k}^{l}\right\rangle}{\sqrt{\Delta_{i} \delta E_{k}}}, \quad \bar{V}_{i n} \equiv \frac{\left\langle X_{i}^{l}|V| \Psi_{n, l}\right\rangle}{\sqrt{\Delta_{i}}}
$$

На основе представления (37) для функции Грина получаем "конечномерное" представление для внемассовой $T$-матрицы

$$
\begin{aligned}
T_{i j}^{l}(E) \approx & V_{i j}-\sum_{n} \frac{\bar{V}_{i n} \bar{V}_{n j}}{E_{n, l}-E}-\sum_{k=1}\left\{\widetilde{V}_{i k} \widetilde{V}_{k j} \ln \left|\frac{E_{k}-E}{E_{k-1}-E}\right|+\right. \\
+ & \left.\left(\widetilde{V}_{i k+1} \widetilde{V}_{k+1 j}-\widetilde{V}_{i k} \widetilde{V}_{k j}\right)\left[1+\frac{\left(E-E_{k}^{*}\right)}{\delta E_{k}} \ln \left|\frac{E_{k}-E}{E_{k-1}-E}\right|\right]\right\}- \\
& -i \pi\left\{\widetilde{V}_{i k_{0}} \widetilde{V}_{k_{0} j}+\left(\widetilde{V}_{i k_{0}+1} \widetilde{V}_{k_{0}+1 j}-\widetilde{V}_{i k_{0}} \widetilde{V}_{k_{0} j}\right) \frac{\left(E-E_{k_{0}}^{*}\right)}{\delta E_{k_{0}}}\right\}
\end{aligned}
$$

при $E \in\left(E_{k_{0}-1}, E_{k_{0}}\right)$.

На массовой поверхности $E_{1}=E_{2}=E$, и тогда в узловых точках $E \equiv \mathcal{E}_{i}^{*}$ из (48) получаем, что

$$
\begin{aligned}
T_{l}(E, E ; E)= & T_{i i}^{l}\left(\mathcal{E}_{i}^{*}\right) \approx V_{i i}-\sum_{n} \frac{\bar{V}_{i n}^{2}}{E_{n, l}-\mathcal{E}_{i}^{*}}-\sum_{k=1}\left\{\widetilde{V}_{i k}^{2} \ln \left|\frac{E_{k}-\mathcal{E}_{i}^{*}}{E_{k-1}-\mathcal{E}_{i}^{*}}\right|+\right. \\
& \left.+\left(\widetilde{V}_{i k+1}^{2}-\widetilde{V}_{i k}^{2}\right)\left[1+\frac{\left(\mathcal{E}_{i}^{*}-E_{k}^{*}\right)}{\delta E_{k}} \ln \left|\frac{E_{k}-\mathcal{E}_{i}^{*}}{E_{k-1}-\mathcal{E}_{i}^{*}}\right|\right]\right\}- \\
& -i \pi\left\{\widetilde{V}_{i k_{0}}^{2}+\left(\widetilde{V}_{i k_{0}+1}^{2}-\widetilde{V}_{i k_{0}}^{2}\right) \frac{\left(\mathcal{E}_{i}^{*}-E_{k_{0}}^{*}\right)}{\delta E_{k_{0}}}\right\} .
\end{aligned}
$$

Преимушеством найденных выражений для матрицы рассеяния на энергетической поверхности и вне ее является то, что в них входят только матричные элементы потенциала взаимодействия в базисе локализованных волновых пакетов. Как будет показано в следуюшем разделе, при разложении таких локализованных пакетов по $L_{2}$-базису из (48) и (49) получаются явные аналитические формулы, которые содержат лишш матричные элементы оператора взаимодействия $V$ по выбранному $L_{2}$-базису. Это, в свою очередь, позволяет свести нахождение операторов рассеяния лишш к простым алгебраическим формулам, содержащим матрицу потенциала в $L_{2}$-базисе в качестве основного элемента.

Можно показать, что найденные конечномерные представления для функции Грина и внемассовой $T$-матрищы сходятся к своим точным значениям при $\Delta_{i} \rightarrow 0$ и $E_{\max } \rightarrow \infty$ $\left(E_{\max }-\right.$ верхняя граница непрерывного спектра, учитьваемого в разложениях $(37),(44)$ и (48)).

Для иллюстрации конечномерной аппроксимации свободной функции Грина приведем выражение для матрицы оператора

$$
\bar{T}=V-V G_{0}^{(+)} V,
$$


отвечающего второму порядку теории возмущений, в “узловых" точках по энергии $E=\mathcal{E}_{i}^{*}$,

$$
\bar{T}_{i}^{l} \equiv\left\langle\Psi_{0}\left(\mathcal{E}_{i}^{*}, l\right)\left|V-V G_{0}^{(+)} V\right| \Psi_{0}\left(\mathcal{E}_{i}^{*}, l\right)\right\rangle,
$$

где оператор Грина $G_{0}^{(+)}$гамильтониана свободного движения можно записать аналогично (37) через СД функций свободного движения, т.е.

$$
\begin{aligned}
\bar{T}_{i}^{l} \approx & V_{i i}-\sum_{k=1}\left\{V_{i k}^{2} \ln \left|\frac{\mathcal{E}_{k}-\mathcal{E}_{i}^{*}}{\mathcal{E}_{k-1}-\mathcal{E}_{i}^{*}}\right|+\right. \\
& \left.+\left(V_{i k+1}^{2}-V_{i k}^{2}\right)\left[1+\frac{\left(\mathcal{E}_{i}^{*}-\mathcal{E}_{k}^{*}\right)}{\Delta_{k}} \ln \left|\frac{\mathcal{E}_{k}-\mathcal{E}_{i}^{*}}{\mathcal{E}_{k-1}-\mathcal{E}_{i}^{*}}\right|\right]\right\}-i \pi V_{i i}^{2}
\end{aligned}
$$

Формулы, полученные в этом пункте, удобны тем, что для расчетов с использованием ПС Д достаточно лишш один раз вычислить матричные элементы (47), а далее все сводится к простым алгебраическим вычислениям. Однако все еше остается открытым вопрос оптимального построения самих С.Д. Желательно также найти аналитический вид матричных элементов (47). Этим вопросам будет посвящен следующий раздел.

\section{3. СОБСТВЕННЫЕ ДИФФЕРЕНЦИАЛЫ \\ И ДИСКРЕТИЗАЦИЯ КОНТИНУУМА В ОСЦИЛЛЯТОРНОМ БАЗИСЕ. ПРИЛОЖЕНИЕ К КОНЕЧНОМЕРНОЙ АППРОКСИМАЦИИ ОПЕРАТОРОВ РАССЕЯНИЯ}

Рассмотрим гамильтониан (1). Будем искать его собственные функции в виде разложения по конечному дискретному осцилляторному базису,

$$
\widehat{\Psi}_{p, l m}(\mathbf{r}) \equiv \widehat{\Psi}_{p, l}(r) Y_{l m}(\hat{\mathbf{r}})=\sum_{n=0}^{N_{\max }} C_{p n}^{l} \Phi_{n l m}(\mathbf{r})
$$

где элементы базиса

$$
\begin{gathered}
\Phi_{n l m}(\mathbf{r}) \equiv|n l m\rangle=R_{n l}(r) Y_{l}^{m}(\hat{\mathbf{r}}), \\
R_{n l}(r)=\sqrt{\frac{2 n !}{r_{\omega}^{3} \Gamma(n+l+3 / 2)}} e^{-\frac{1}{2} r^{2}}\left(\frac{r}{r_{\omega}}\right)^{l} L_{n}^{l+\frac{1}{2}}\left(\left(\frac{r}{r_{\omega}}\right)^{2}\right)
\end{gathered}
$$

суть собственные функции гармонического осциллятора с частотой $\omega$. В формуле (54) параметр $r_{\omega}$ представляет собой характерный радиус осциллятора

$$
r_{\omega}=\sqrt{\frac{\hbar}{m \omega}}
$$

Задача вычисления коэффициентов $C_{p n}^{l}$ сводится к диагонализации матрицы

$$
\left\|\left\langle n l m|H| n^{\prime} l m\right\rangle\right\|, \quad n, n^{\prime} \leqslant N_{\max }
$$


В результате мы получаем набор собственных функций $\left\{\widehat{\Psi}_{p, l}(r)\right\}_{p=1}^{N_{\max }}$ и собственных значений $\left\{E_{p}^{*}\right\}_{p=1}^{N_{\max }}$, обладаюших следуюшими свойствами:

$$
\left\langle\widehat{\Psi}_{k, l} \mid \widehat{\Psi}_{p, l}\right\rangle=\delta_{k, p}, \quad\left\langle\widehat{\Psi}_{k, l}|H| \widehat{\Psi}_{p, l}\right\rangle=E_{p}^{*} \delta_{k, p} .
$$

Если гамильтониан $H$ имеет конечный дискретный, а также полный непрерывный спектр, то несколько низколежащих собственных значений $\left(p<p_{0}\right)$ будут отвечать дискретному, остальные - непрерывному спектру $H$. Функции $\widehat{\Psi}_{p, l}\left(p \geqslant p_{0}\right)$ будут практически совпадать с точными функциями рассеяния $\Psi\left(E_{p}^{*}, l ; r\right)$ в ограниченной области $r<R_{\max }^{\text {осц }}$ [5]. Таким образом, функции $\widehat{\Psi}_{p, l}$ по своим свойствам сходны с функциями $Y_{p}^{l}$, отвечаюшими разбиению $0=E_{p_{0}}<\cdots<E_{p}<\cdots$, в котором $\left(E_{p-1}+E_{p}\right) / 2=$ $E_{p}^{*}$. Тогда на новом "конечном носителе", отвечаюшем использованному $L_{2}$-базису $(r<$ $\left.\min \left(R_{\max }^{\text {ocu }}, R_{\max }\right)\right)$, можно записать следуюшее равенство:

$$
\widehat{\Psi}_{p, l}(r)=\sum_{n=0}^{N_{\max }} C_{p n}^{l} R_{n l}(r) \approx Y_{p}^{l}(r),
$$

и во всех формулах предыдушего раздела заменить С Д на функции, представленные в осцилляторном базисе. Следует оговориться, что при такой замене вместо бесконечного набора $\left\{Y_{p}^{l}\right\}_{p=p_{0}}^{\infty}$ будет использоваться конечньй набор состояний. При этом мы исследуем сходимость аппроксимируемых величин (элементов $T$-матрицы) к своим точным значениям с увеличением числа базисных функций $N_{\max }$ (более грубая конечномерная аппроксимация операторов рассеяния вместе с соответствуюшими иллюстрациями описана в наших предыдущих работах [4], [5]).

Для С.Д функций свободного рассеяния (42) имеются две возможности. Первая состоит в замене свободных волновых пакетов $X_{k}^{l}$ на соответствуюшие функции $\widehat{\Psi}_{p, l}^{0}$, полученные при диагонализации матрицы гамильтониана свободного движения $H_{0}$ в осцилляторном базисе. Вторая возможность состоит в том, чтобы использовать для функций свободного движения $\Psi_{0}(E, l ; r)$ хорошо известные разложения по осцилляторным функциям [7], а именно,

$$
\Psi_{0}(E, l ; r)=\sum_{n=0}^{\infty} D_{n}^{l}(E) R_{n l}(r)
$$

где

$$
D_{n}^{l}(E)=(2 E)^{\frac{1}{4}}(-1)^{n} \sqrt{\frac{2 n !}{(\sqrt{\hbar \omega})^{3} \Gamma\left(n+l+\frac{3}{2}\right)}}\left(\sqrt{\frac{2 E}{\hbar \omega}}\right)^{l} e^{-\frac{E}{\hbar \omega}} L_{n}^{l+\frac{1}{2}}\left(\frac{2 E}{\hbar \omega}\right) .
$$

Можно теперь построить разбиение непрерывного спектра $\left\{\mathcal{E}_{k}\right\}$ и состояния $X_{k}^{l}$, ограничиваясь в разложении (59) конечным числом слагаемых $N \leqslant N_{\max }$ (или просто непосредственно подставить в формулу (39) разложение (59)).

Так или иначе, СД функций свободного рассеяния можно представить в виде, аналогичном (58),

$$
X_{k}^{l}(r) \approx \sum_{n=0}^{N_{\max }} A_{k n}^{l} R_{n l}(r)=\widehat{\Psi}_{k, l}^{0}(r)
$$


В результате для матричных элементов (47) получим

$$
\begin{gathered}
V_{i j}=\frac{\left\langle X_{i}^{l}|V| X_{j}^{l}\right\rangle}{\sqrt{\Delta_{i} \Delta_{i}}}=\frac{1}{\sqrt{\Delta_{i} \Delta_{j}}} \sum_{n, n^{\prime}=0}^{N_{\max }} A_{i n}^{l} A_{j n^{\prime}}^{l} V_{n, n^{\prime}}^{l}, \\
\widetilde{V}_{i k}=\frac{1}{\sqrt{\Delta_{i} \delta E_{k}}} \sum_{n, n^{\prime}=0}^{N_{\max }} A_{i n}^{l} C_{k n^{\prime}}^{l} V_{n, n^{\prime}}^{l}, \quad \widehat{V}_{i n}=\frac{1}{\sqrt{\Delta_{i}}} \sum_{k, k^{\prime}=0}^{N_{\max }} A_{i k}^{l} C_{n k^{\prime}}^{l} V_{k, k^{\prime}}^{l},
\end{gathered}
$$

причем вместо точных волновых функций $\left\{\Psi_{n, l m}\right\}$, отвечаюших связанным состояниям, следует использовать функции $\widehat{\Psi}_{p, l}$ с $p<p_{0}$. В выражениях (62) появляются основные элементы, с которыми будут проводиться все вычисления операторов рассеяния - элементы матрицы потенциала взаимодействия в осцилляторном базисе

$$
V_{n, n^{\prime}}^{l} \equiv\left\langle n l m|V| n^{\prime} l m\right\rangle, \quad n, n^{\prime} \leqslant N_{\max } .
$$

Можно теперь переписать формулы для $T$-матрицы, используя матричные элементы (63), в представлении функций гармонического осциллятора.

В описанном подходе все еше остался открытым вопрос нахождения точек разбиения непрерывного спектра $E_{p}$, отвечающего СД $Y_{p}^{l}$, так как при построении состояний $\widehat{\Psi}_{p, l}$ известны только средние точки $E_{p}^{*}$. При этом известно пороговое положение энергии $E_{0}=0$. Переобозначая $E_{0} \rightarrow E_{p_{0}}$, можно по значениям "средних" энергий $E_{p}^{*}$ построить далее узловые точки $E_{p}$, следуя простому алгоритму

$$
E_{p_{0}}=0, \quad E_{p}=2 E_{p}^{*}-E_{p-1}, \quad p=p_{0}+1, \ldots, N_{\max } .
$$

Соответственно для ширин энергетических интервалов будем иметь

$$
\delta E_{p}=E_{p}-E_{p-1} .
$$

Аналогично строятся и узловые точки $\mathcal{E}_{k}$ для состояний $\widehat{\Psi}_{p, l}^{0}$.

\section{4. ЧИСЛЕННЫЕ ИЛЛЮСТРАЦИИ}

В этом разделе мы изучим на серии конкретных примеров точность и скорость сходимости конечномерных приближений для функции Грина и $T$-матришы при использовании в качестве $L_{2}$-базиса набора волновых функций гармонического осциллятора. Подчеркнем при этом, что наш подход кардинально отличается от многочисленных использованных ранее схем разложения волновых функций рассеяния по функциям гармонического осциллятора [7]-[15], хотя некоторые общие элементы и присутствуют.

Известные нам работы в этой области можно разделить на две части. Авторы работ [7], [9], [10] вводят так называемое осцилляторное представление для волновых функций рассеяния вместо координатного. При этом функции, а также операторы [10] рассеяния раскладываются в осцилляторные ряды при одной фиксированной әнергии. Далее, используя специфические свойства выбранного базиса (представление оператора кинетической энергии в виде трехдиагональной матрицы), в осцилляторном представлении производится "сшивка" осцилляторных разложений со свободными решениями 
вне области действия потенциала [7], [9], [10]-[12]. Главное отличие предлагаемого нами подхода от вьшеизложенного состоит в том, что осцилляторный базис является в нашем случае лишь удобным частным примером $L_{2}$-базиса разложения (с равным успехом можно использовать любой другой дискретный базис). Еще одно отличие состоит в том, что мы строим конечномерные представления для операторов рассеяния сразу при всех энергияx, для чего требуется всего одна диагонализация матрицы гамильтониана.

Предлагаемый нами подход ближе к методам второго направления, развитым в основном в работах по молекулярной физике [13]-[15]. Там нахождение конечномерных аппроксимаций для функции Грина основывается на построении специальных квадратур для спектральных интегралов (метод квадратур Стилтьеса-Чебышева). В нашем подходе подобные выражения строятся с помощью использования нового формализма СД функций рассеяния. Отметим также, что в наших предыдуших работах [4], [5] использовался похожий метод нахождения аппроксимации операторов рассеяния в осцилляторном базисе, основанный, однако, на феноменологических представлениях.

И, наконец, самое главное. Поскольку мы развиваем конечномерные аппроксимации для операторов рассеяния на конечном носителе, данный подход непосредственно обобшается на многочастичный случай (с использованием уравнений Фаддеева-Якубовского) в отличие от известных методов разложения волновых функций рассеяния по осцилляторному базису, где такое обобшение до сих пор не было выполнено, несмотря на многочисленные попытки.

4.1. Взаимодействие в форме локального потенциала. В качестве первого примера рассмотрим сходимость аппроксимации в базисе С.Д функций свободного движения $X_{i}^{l}$ борновского члена $T$-матрицы $\widehat{V}(E) \equiv\left\langle\Psi_{0}(E, l)|V| \Psi_{0}(E, l)\right\rangle$ в случае локального потенциала гауссова вида

$$
V(r)=-V_{0} e^{-\gamma r^{2}}
$$

когда имеется простая аналитическая формула

$$
\widehat{V}(E)=-\frac{V_{0}}{E} \frac{k^{2}}{4 \gamma} e^{-\frac{k^{2}}{2 \gamma}} I_{l+\frac{1}{2}}\left(\frac{k^{2}}{2 \gamma}\right), \quad k=\sqrt{\frac{2 m E}{\hbar^{2}}},
$$

где $I_{l+1 / 2}$ - модифицированная функция Бесселя.

Согласно (43) имеем

$$
\widehat{V}\left(\mathcal{E}_{i}^{*}\right) \approx V_{i i}=\frac{\left\langle X_{i}^{l}|V| X_{i}^{l}\right\rangle}{\Delta_{i}}
$$

Вместо С Д $X_{i}^{l}(r)$ функций свободного движения $\Psi_{0}(E, l ; r)$ будем использовать аналогичные по смыслу функции $\widehat{\Psi}_{p, l}^{0}(r)$, полученные после диагонализации матрицы свободного гамильтониана $H_{0}=\mathbf{p}^{2} / 2 m$ на конечном осцилляторном базисе. При этом удобно использовать два безразмерных параметра

$$
v_{0}=\frac{V_{0}}{\hbar \omega} \quad \text { и } \quad q=\sqrt{\gamma} r_{\omega},
$$


$\widehat{V}(E)$
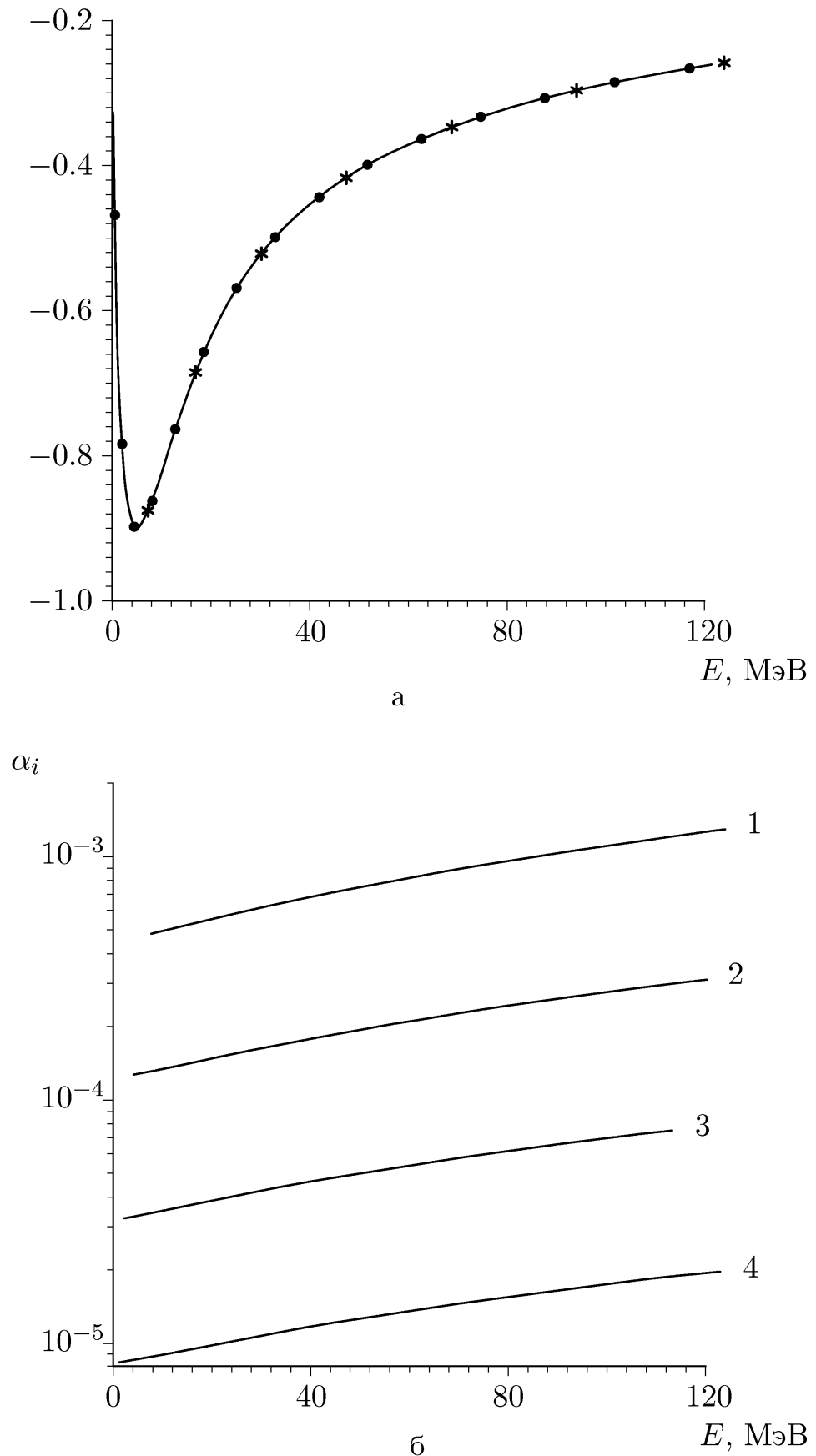

Рис. 1 
связанные с безразмерным параметром силы потенциала [16] соотношением

$$
\xi=\frac{\hbar^{2} \gamma}{m V_{0}}=\frac{q^{2}}{v_{0}}
$$

При использовании функций $\widehat{\Psi}_{p, l}^{0}$ в виде $(61)$ для расчета матричных элементов $V_{i j}$ согласно (62) будем использовать простые аналитические формулы

$$
V_{i j}=\frac{1}{\sqrt{\Delta_{i} \Delta_{j}}} \sum_{n, n^{\prime}=0}^{N_{\max }} A_{i n}^{l} A_{j n^{\prime}}^{l} V_{n, n^{\prime}}^{l}
$$

где

$$
V_{n, n^{\prime}}^{l}=-V_{0}\left[\frac{\left(l+\frac{3}{2}\right)_{n}\left(l+\frac{3}{2}\right)_{n^{\prime}}}{n ! n^{\prime} !}\right]^{\frac{1}{2}} \frac{\left(q^{2}\right)^{n+n^{\prime}}}{\left(q^{2}+1\right)^{n+n^{\prime}+l+\frac{3}{2}}}{ }_{2} F_{1}\left(-n,-n^{\prime} ; l+\frac{3}{2} ; \frac{1}{q^{4}}\right) .
$$

Расчеты проводились для значений $V_{0}=-41.47 \mathrm{MэB}, \gamma=0.2^{-2}, m=1$ а.е.м. (что отвечает $\xi=0.2)$ и $l=0$. Параметр $q$, относяшийся к выбору радиального масштаба базиса, является свободным и подбирается оптимальным образом, чтобы обеспечить наиболее быструю сходимость величин $V_{i i}$ с увеличением размеров базиса $N_{\max }$.

На рис. 1а приведены зависимости величин $V_{i i}$ от энергии в сравнении с точными значениями $\widehat{V}(E)$ (сплошная кривая) для $N_{\max }=20(*)$ и $N_{\max }=160 \bullet$. Хорошо видно, что даже при использовании небольшого базиса разложения с $N_{\max }=20$ для аппроксимации свободных волновых пакетов $X_{i}^{l}$ получается весьма точная аппроксимация борновского члена в большом интервале энергий.

На рис. 16 приведены зависимости от энергии относительных погрешностей величины $\widehat{V}(E)$

$$
\alpha_{i}=\frac{\left|\widehat{V}\left(\mathcal{E}_{i}^{*}\right)-V_{i i}\right|}{\left|\widehat{V}\left(\mathcal{E}_{i}^{*}\right)\right|}
$$

для разных $N_{\max }\left(N_{\max }=20\right.$ для кривой 1,40 - для кривой 2,80 - для кривой 3,160 для кривой 4). При этом параметр $q$ выбран одинаковым для всех кривых. Видно, что относительная погрешность, возникаюшая при замене базиса плоских волн соответствующими СД, не превосходит $0.1 \%$ даже при минимальных размерах базиса. Таким образом, даже в случае плоских волн использование волновых пакетов в осцилляторном базисе представляет большие удобства, поскольку позволяет работать при всех вычислениях только с матрицей потенциала в этом базисе.

В качестве второго примера рассмотрим элементы $T$-матрицы на массовой поверхности для того же потенциала (66). При этом исследовалась “упрощенная" схема нахождения $T_{l}(E)$. Вместо осцилляторных разложений для свободных пакетов $X_{i}^{l}$ в этом случае использовались точные функции свободного движения $\Psi_{0}(E, l ; r)$, а ПС Д-аппроксимация применялась только для оператора Грина $G_{l}^{(+)}(E)($ см. $(37))$. При этом в качестве функций $\left|Y_{i}^{l}\right\rangle$ используются собственные функции, полученные при диагонализации матрицы гамильтониана $H$ в осцилляторном базисе. Величины $T_{l}(E)$ вычисляются по формуле

$$
T_{l}(E)=\left\langle\Psi_{0}(E, l)|V| \Psi_{0}(E, l)\right\rangle-\left\langle\Psi_{0}(E, l)\left|V G^{(+)} V\right| \Psi_{0}(E, l)\right\rangle .
$$


$\operatorname{Re} T_{0}$

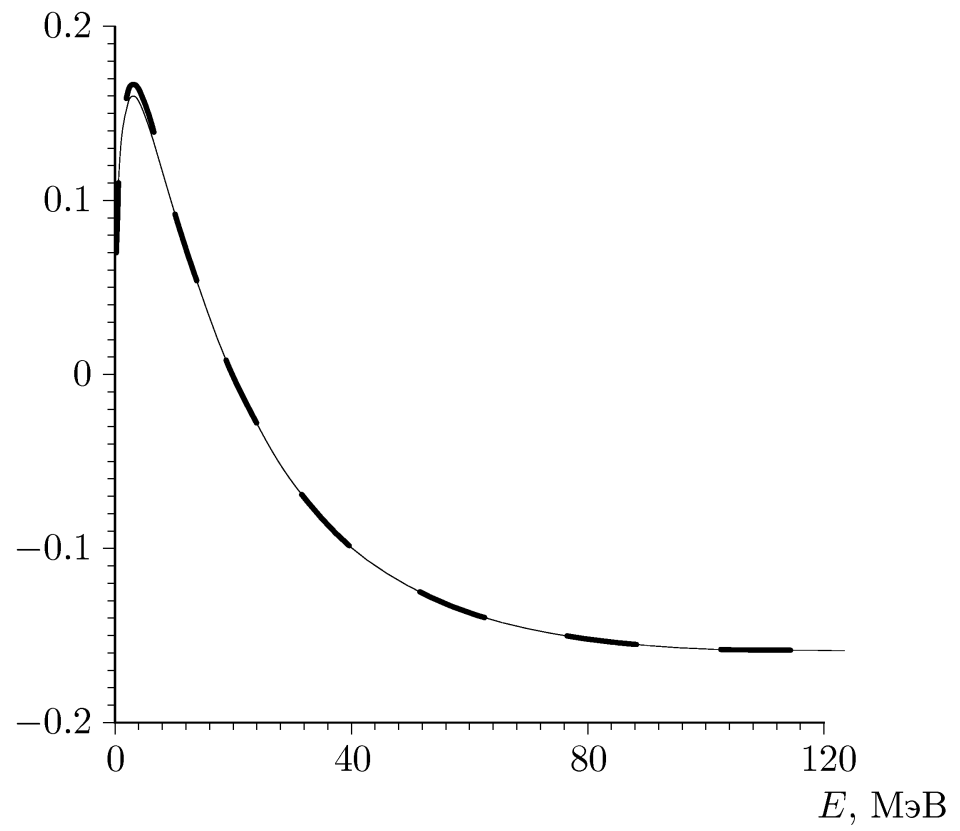

a

$\operatorname{Im} T_{0}$

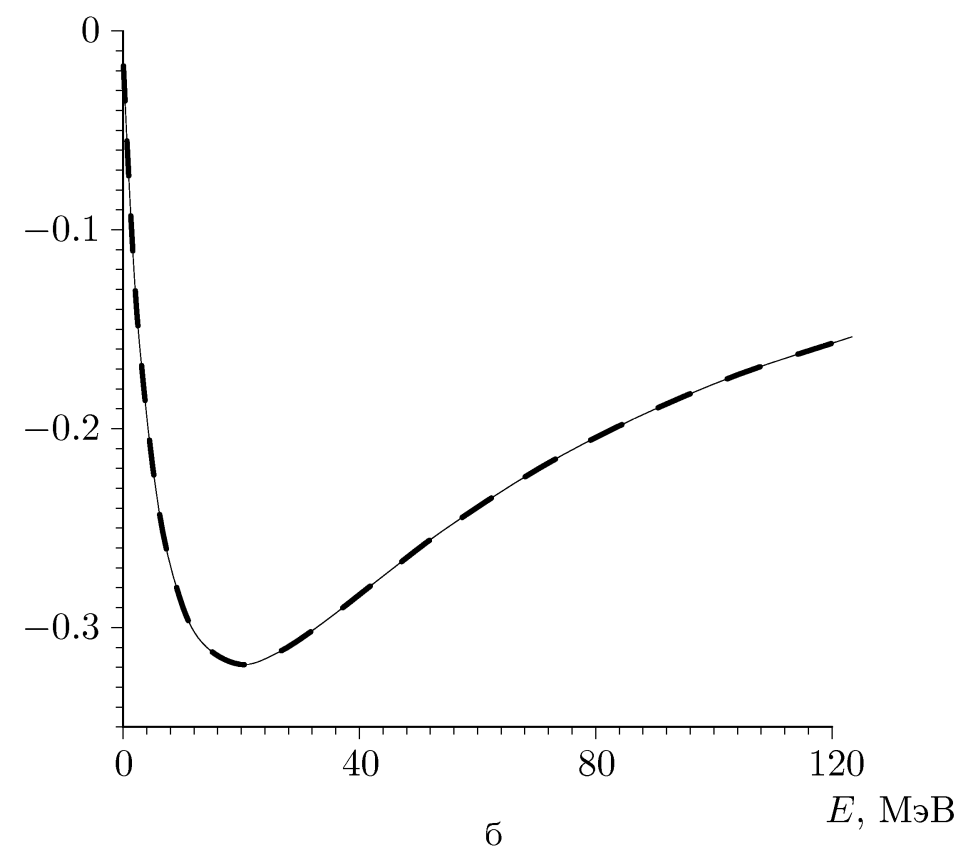

Рис. 2 
Первое слагаемое в (70) представляет собой элемент матрицы рассеяния в борновском приближении (67). Второе интегральное слагаемое в (70) вычисляется на основе (37), где вместо $\left|Y_{p}^{l}\right\rangle$ следует поставить элементы $\left\langle\Psi_{0}(E ; l)|V| \widehat{\Psi}_{p, l}\right\rangle$, которые также имеют аналитический вид [5].

На рис. 2 приведены зависимости от энергии действительных (а) и мнимых (б) частей приближенных функций $T(E)$ соответственно для разных размеров $\left(N_{\max }=40-\right.$ штриховая линия, $N_{\max }=150$ - сплошная линия) осцилляторного базиса в случае $l=0$. Совершенно очевидно, что уже при размерности базиса $N_{\max }=40$ достигается полная сходимость как для действительной, так и для мнимой частей матрицы рассеяния. Очень ясное представление о характере сходимости результатов к точным можно составить на основе изучения точности выполнения условия унитарности для приближенной парциальной $S$-матрицы $S_{l}(E)$ (как известно, в случае чисто потенциального рассеяния $\left.\left|S_{l}(E)\right|=1=\sqrt{\left(\operatorname{Re} S_{l}\right)^{2}+\left(\operatorname{Im} S_{l}\right)^{2}}\right)$.

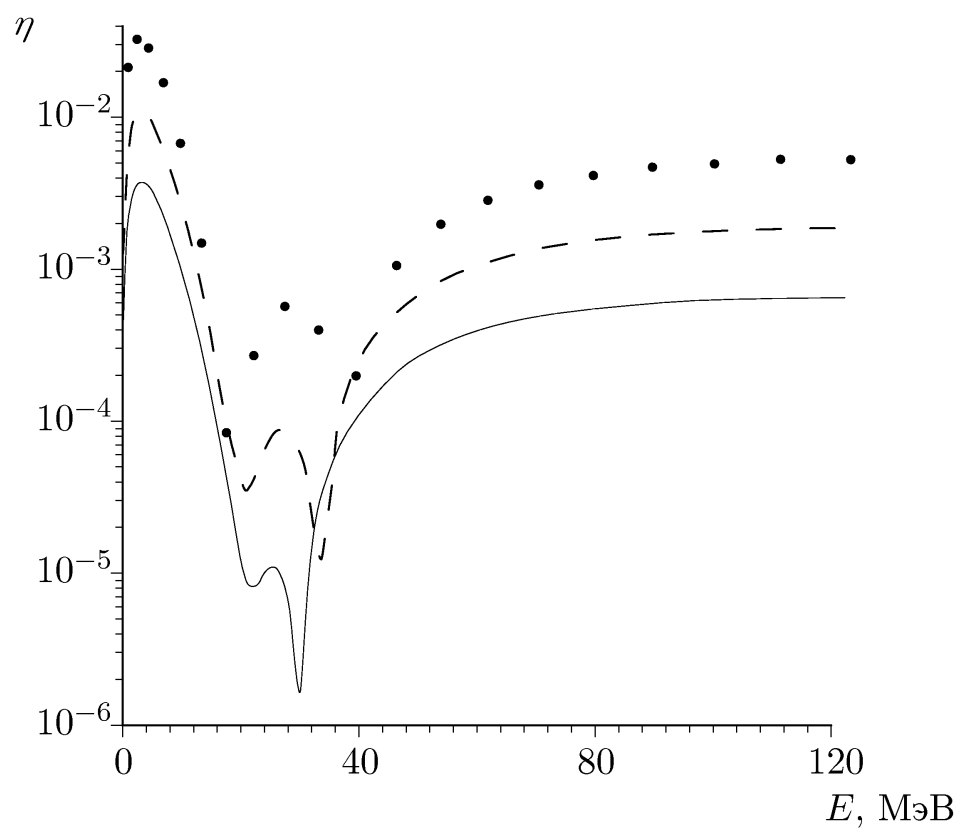

Рис. 3

На рис. 3 представлена зависимость от энергии величины $\eta=|1-| S_{0}(E)||$, вычисленной с помощью “дискретизованной” $T$-матрицы на массовой поверхности (мы использовали стандартное соотношение между $T$ - и $S$-матрицами: $S_{l}(E)=1-2 \pi i T_{l}(E)$ ). На этом рисунке точки $\bullet$ соответствуют $N_{\max }=40$, штриховая линия $-N_{\max }=80$, сплошная линия $-N_{\max }=150$. Отличие модуля $S$-матрицы от единицы обусловлено погрешностями аппроксимации. Хорошо видно, что погрешность приближенной $T$-матрицы быстро убывает с увеличением размерности используемого базиса.

Подчеркнем, что парциальная функция Грина, а также $T$-матрица при всех энергиях на массовой поверхности и вне ее находятся в нашем подходе из однократной простой ди- 
агонализации матрицы гамильтониана в выбранном $L_{2}$-базисе. Поскольку этот расчет весьма прост, развитый метод дискретизации континуума приводит к очень быстрой и экономной вычислительной схеме.

4.2. Взаимодействие в форме нелокального потенциала. Для иллюстрации эффективности и универсальности предлагаемого подхода мы рассмотрим также вычисление $T$-матрицы для нелокального потенциала с ядром следующего вида:

$$
V\left(\mathbf{r}, \mathbf{r}^{\prime}\right)=-V_{0} U\left(\frac{1}{2}\left|\mathbf{r}+\mathbf{r}^{\prime}\right|\right) P\left(\left|\mathbf{r}-\mathbf{r}^{\prime}\right|\right),
$$

где

$$
U(x)=e^{-\gamma x^{2}}, \quad P(y)=\frac{e^{-\frac{y^{2}}{\beta^{2}}}}{\pi^{\frac{3}{2}} \beta^{3}} .
$$

Следует подчеркнуть, что в нашем подходе рассмотрение нелокального потенциала в принципе столь же просто, как и случай локального взамодействия. Меняются только выражения для борновского члена и для элементов $\left\langle\Psi_{0}|V| \widehat{\Psi}_{p, l}\right\rangle$ (отметим, что для нелокального потенциала уравнение Шредингера из обычного дифференциального преврашается в интегро-дифференциальное).

В случае нелокального потенциала вида (71) удобно ввести три безразмерных параметра

$$
v_{0}=\frac{V_{0}}{\hbar \omega}, \quad q=\sqrt{\gamma} r_{\omega}, \quad b=\frac{\beta}{r_{\omega}} .
$$

Для борновского члена (при $l=0)$ получается следуюшая формула:

$$
\left\langle\Psi_{0}(E, 0)|V| \Psi_{0}(E, 0)\right\rangle=-\frac{V_{0}}{E} \frac{1}{(\sqrt{D} \beta)^{3}} \sqrt{\frac{k^{2} D}{\pi A W}} e^{-\frac{k^{2}}{2 A}} \operatorname{sh} \frac{k^{2} D}{4 A W},
$$

где

$$
D=\frac{2}{\beta^{2}}-\frac{\gamma}{2}, \quad W=\frac{\gamma}{4}+\frac{1}{\beta^{2}}, \quad A=W-\frac{D^{2}}{4 W} .
$$

Для матричных элементов $\left\langle\Psi_{0}|V| \widehat{\Psi}_{p, l}\right\rangle$ аналитический расчет [17] приводит к выражению

$$
\left\langle\Psi_{0}|V| \widehat{\Psi}_{p, l}\right\rangle=-V_{0} B_{p, l}
$$

где

$$
\begin{aligned}
B_{p, l}\left(k, q^{2}, b^{2}\right)= & \frac{1}{\sqrt{2 E}}\left(\frac{k r_{\omega}}{2 u}\right)^{l+\frac{3}{2}}\left(\frac{d}{2}\right)^{l} \frac{1}{b^{3}} e^{-\frac{\left(k r_{\omega}\right)^{2} w}{4 u}} \times \\
& \times \sum_{n=0}^{N_{\max }} C_{p n}^{l}\left[\frac{2 n !}{\Gamma\left(n+l+\frac{3}{2}\right)}\right]^{\frac{1}{2}}\left(\frac{v}{u}\right)^{n} L_{n}^{l+\frac{1}{2}}\left(\frac{\left(k r_{\omega}\right)^{2} d^{2}}{16 u v}\right) .
\end{aligned}
$$

Здесь введены обозначения

$$
\begin{aligned}
w & =\frac{q^{2}}{4}+\frac{1}{b^{2}}, & d & =\frac{2}{b^{2}}-\frac{q^{2}}{2}, \\
u & =\left(w+\frac{1}{2}\right)(w-1)-\frac{d^{2}}{4}, & v & =\left(w-\frac{1}{2}\right)(w-1)-\frac{d^{2}}{4} .
\end{aligned}
$$



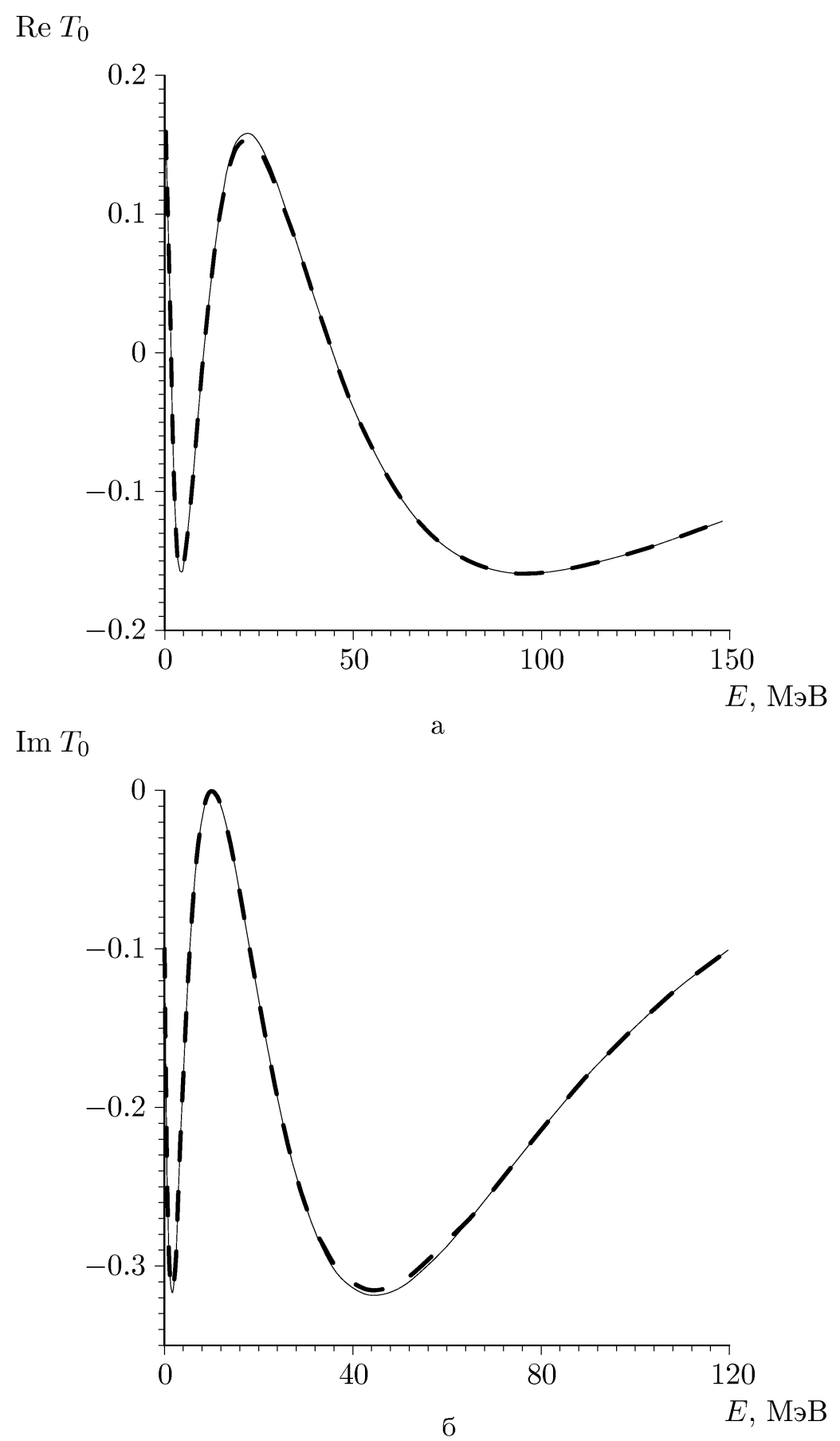

Рис. 4 
Расчеты проводились для следуюших частных значений параметров потенциала (71):

$$
V_{0}=-69.84 \mathrm{Mэ} \mathrm{B}, \quad \gamma=0.1 \Phi^{-2}, \quad \beta=0.85 \Phi^{-2} .
$$

На рис. 4 представлены зависимости действительных (а) и мнимых (б) частей паршиальной $T$-матришы на массовой поверхности $T_{0}(E)$ при $l=0$ (штриховая линия отвечает $N_{\max }=40$, сплошная $-N_{\max }=150$ ). Снова видно, что скорость сходимости на выбранном базисе для нелокального потеншиала того же порядка величины, что и для локального, т.е. реального увеличения сложности вычислений не происходит.

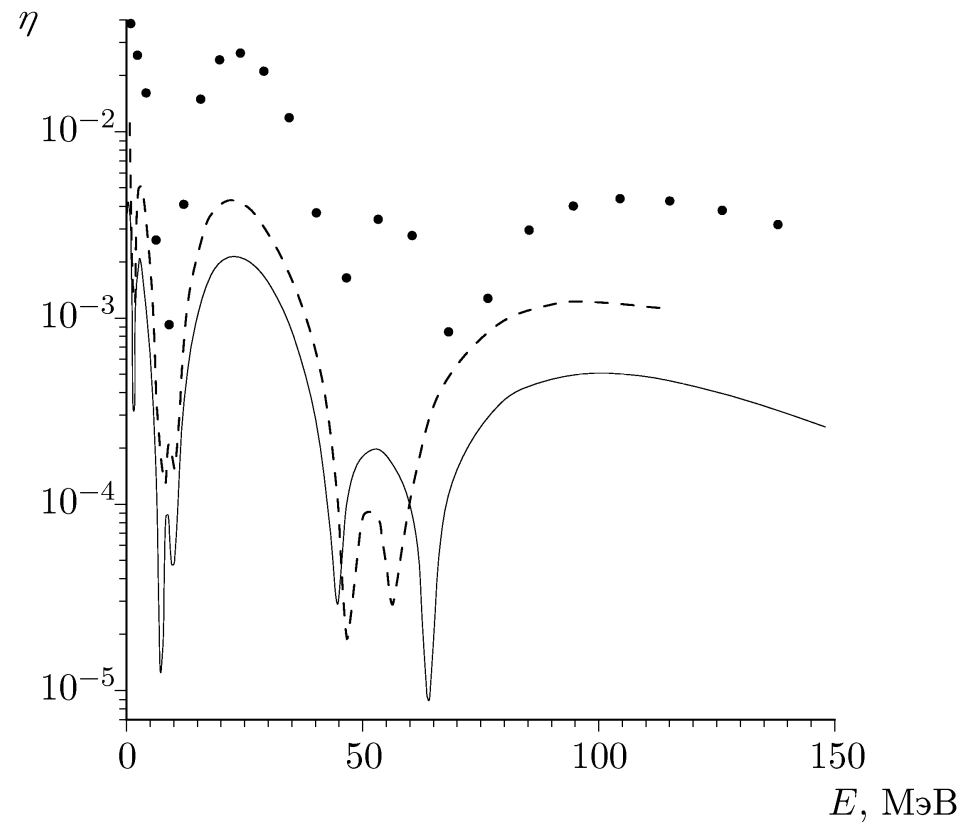

Рис. 5

На рис. 5 представлена зависимость от энергии величины $\eta=|1-| S_{0}(E)||$, характеризуюшей нарушение условия унитарности приближенной $S$-матрицы (как и на рис. 3 , точки $\bullet$ соответствуют $N_{\max }=40$, штриховая линия $-N_{\max }=80$, сплошная линия $\left.N_{\max }=150\right)$. Из сравнения с рис. 3 можно сделать вывод, что сходимость для локального потенциала в среднем является такой же, как и для нелокального.

В работе [5] была показана применимость развитого подхода также и в случае комплексного потенциала взаимодействия. Таким образом, этот подход является вполне универсальным для широкого класса взаимодействий, встречаюшихся в атомной, молекулярной и ядерной физике.

\section{5. ЗАКЛЮЧЕНИЕ}

В данной работе на основе систематического использования представления С Дв квантовой теории рассеяния были получены удобные конечномерные представления для основных операторов теории рассеяния вне массовой поверхности и на ней. Последующее разложение С Д на "конечном носителе" через $L_{2}$-базис позволило найти удобные 
аналитические формулы для основных операторов. При этом для локального и нелокального взаимодействий достаточна однократная диагонализация матрицы соответствуюшего гамильтониана в подходяшем $L_{2}$-базисе, что позволяет найти внемассовую $T$-матрицу и функцию Грина сразу для всех энергий.

Таким образом, развитая схема, основанная на дискретизации континуума, приводит к чрезвычайно быстрому и экономному алгоритму нахождения операторов теории рассеяния. Предложенный подход естественным образом может быть обобщен на задачу рассеяния трех и большего числа частиц с использованием уравнений Фаддеева-Якубовского. Развитая схема дискретизации континуума может иметь многочисленные приложения в атомной и ядерной физике, а также в физике твердого тела.

Благодарности. Работа выполнена при поддержке Российского фонда фундаментальных исследований (грант № 01-02-16621).

\section{Список литературы}

[1] С. П. Меркурьев, Л. Д. Фаддеев. Квантовая теория рассеяния для систем нескольких частиц. М.: Наука, 1985.

[2] R. Y. Rasoanaivo, G. H. Rawitscher. Phys. Rev. C. 1989. V. 39. P. 1709.

[3] A. Мессиа. Квантовая механика. М: Наука, 1978.

[4] О. А. Рубцова, В. И. Кукулин. ЯФ. 2001. Т. 64. С. 1769

[5] О. А. Рубцова, В. И. Кукулин. ЯФ. 2001. Т. 64. С. 1882.

[6] Р. Ньютон. Теория рассеяния волн и частиц. Пер. с англ. М.: Мир, 1969.

[7] А.М. Широков, Ю.Ф. Смирнов. ТМФ. 1998. Т. 117. С. 227.

[8] Г. Ф. Филиппов, И. П. Охрименко. ЯФ. 1980. Т. 32. С. 932; Г. Ф. Филиппов. ЯФ. 1981. T. 33. C. 928

[9] Ю.И. Нечаев, Ю. Ф. Смирнов. ЯФ. 1982. Т. 35. С. 1385.

[10] H. A. Yamani, M.S. Abdelmonem. J. Phys. B. 1997. V. 30. P. 1633.

[11] H. A. Yamani, L. Fishman. J. Math. Phys. 1975. V. 16. P. 410.

[12] J. T. Broad. Phys. Rev. A. 1978. V. 18. P. 1012.

[13] C. T. Corcoran, P. W. Langhoff. Math. Phys. 1977. V. 18(4). P. 651.

[14] J. R. Winick, W. P. Reinhardt. Phys. Rev. A. 1978. V. 18. P. 910.

[15] I. Cacelli, V. Carravetta, A. Rizzo. J. Chem. Phys. 1993. V. 98. P. 8742.

[16] А.И. Базь, Я. Б. Зельдович, А. М. Переломов. Рассеяние, реакции и распады в нерелятивистской квантовой механике. М.: Наука, 1971.

[17] А. П. Прудников, Ю. А. Брычков, О. И. Маричев. Интегралы и ряды. Специальные функции. М.: Наука, 1983. 